\title{
2,3-diketopirrolidines as perspective building blocks for Medicinal Chemistry: synthesis and properties
}

\section{S. Bondarenko, A. Fedorchenko, D. Volochnyuk, S. Ryabukhin}

\section{Introduction and Aim}

The shifting from "traditional" heteroaromatic design concepts in modern Medicinal Chemistry leads to increasing complexity of the synthesis of medicinally relevant molecules. One of the best way for solving this problem is to apply in the synthesis the robust well-known procedures and achieves complexity by using the sophisticated, commercially available (if it is possible), building blocks. Therefore, the interest in 3D-shaped building blocks with high fractions of $F(s p 3)$-hybridized carbon atoms continuously growth and now its synthesis becomes a part of the industry. Therefore, the procedures, which can effectively lead to the formation of targeted products in 10-100 grams scale become more and more demanded.

We focused our attention on the elaboration of the effective multigram scale procedure for the synthesis of diverse 4-substituted 2,3-diketopyrrolidines. These ketones are a very rare class of organic compounds. For today there are only a few methods for obtaining such compounds in milligram scales. On the other hand these products have been perspective building blocks for Medicinal

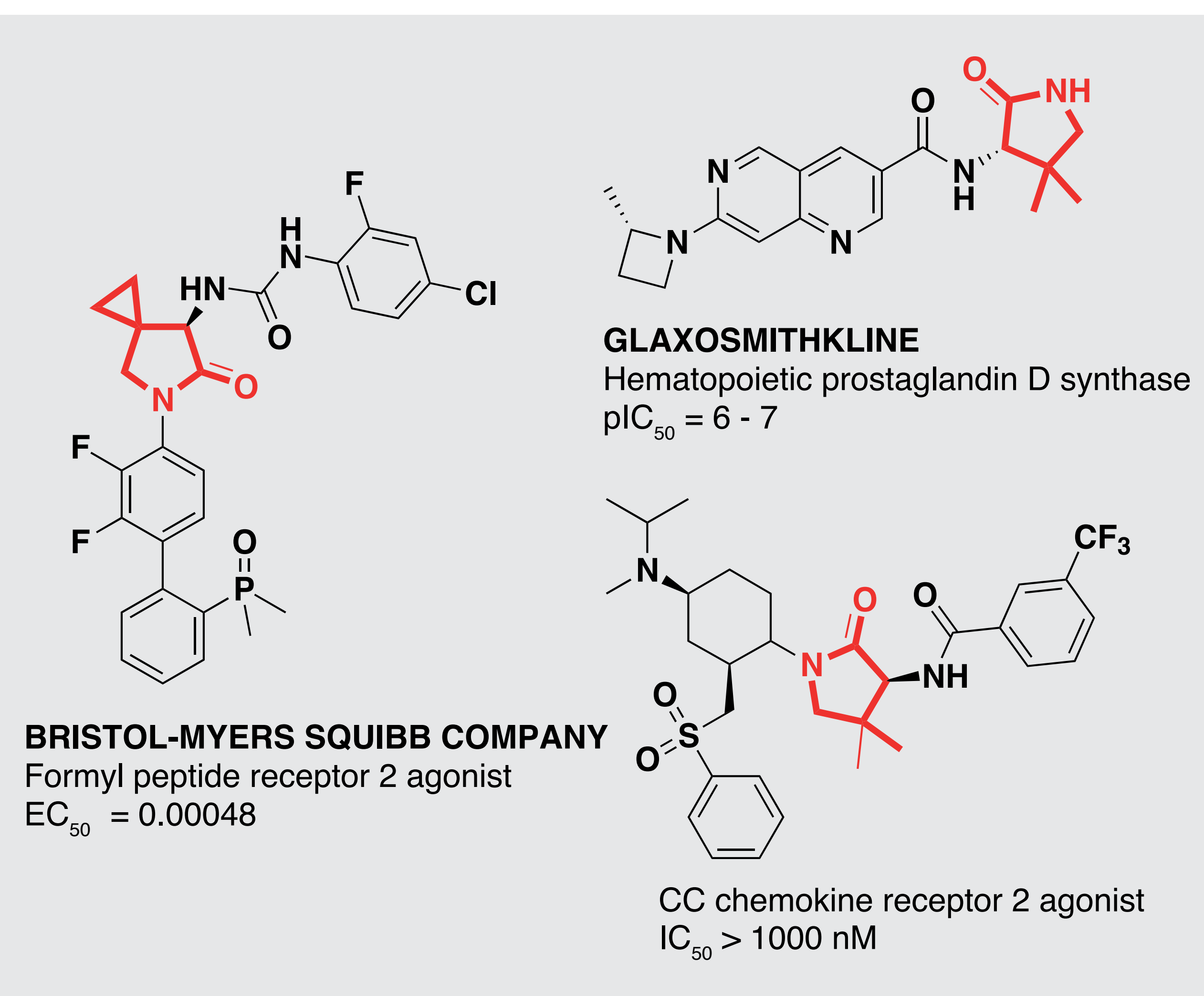
Chemistry needs.

The synthetic methodology for the obtaining of 2,3-diketopyrrolidines is validated and optimized. The advantages and scope and limitations of the procedure are determined.

Synthesis

$$
\text { (n) }
$$
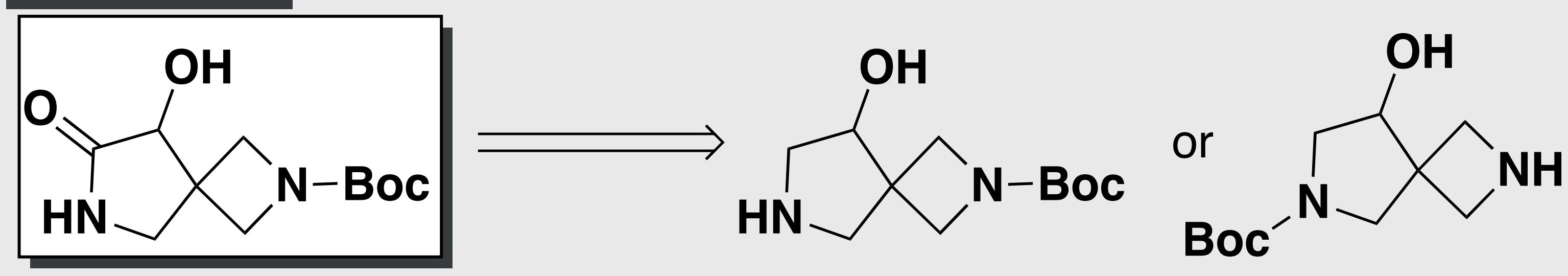

\section{Results}

$$
=0
$$

EN300-247628<smiles>O=C1CC2(CC[R16]([O-])C2)CN1</smiles>

EN300-311561<smiles>CC1(C)CNC(=O)C1N</smiles>

EN300-309434

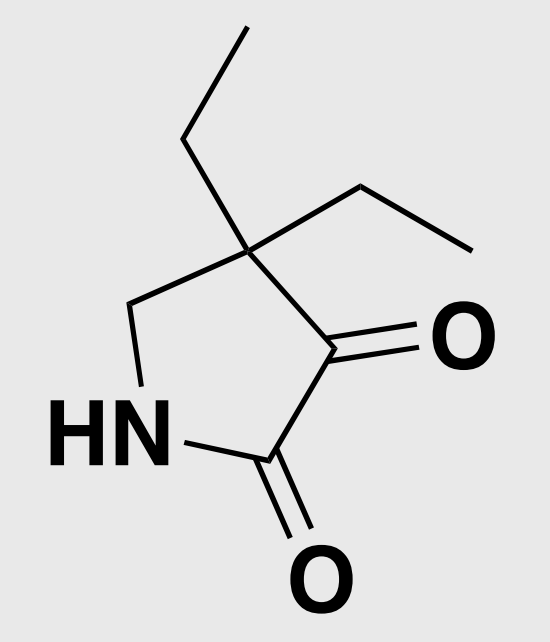

EN300-26666758

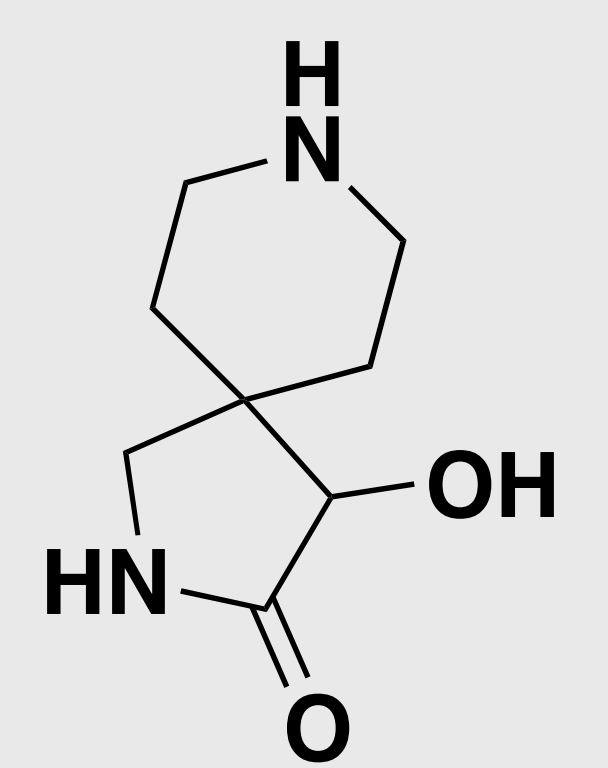

EN300-6478435<smiles>NC1C(=O)NCC12CC2</smiles>

EN300-784919<smiles>O=C1NCC(c2ccccc2)C1=O</smiles>

EN300-6497790<smiles>CC(C)(C)OC(=O)C1CCC2(CC1)CNC(=O)C2N</smiles>

EN300-311560

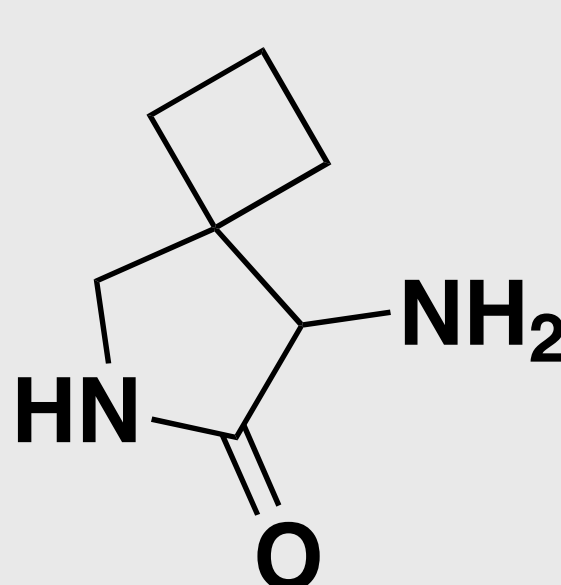

EN300-784921<smiles>O=C1NCC2(CC2)C1=O</smiles>

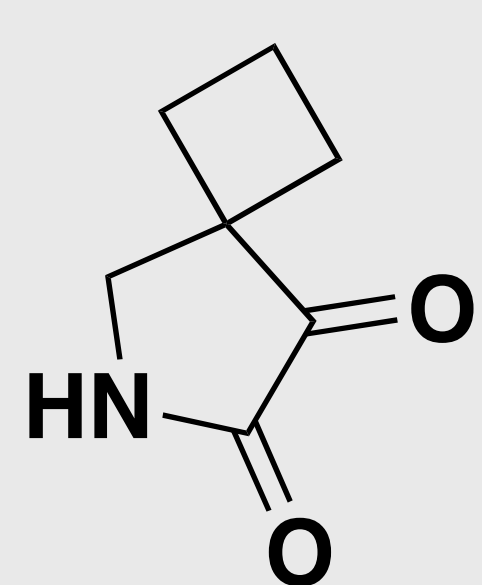

EN300-311562

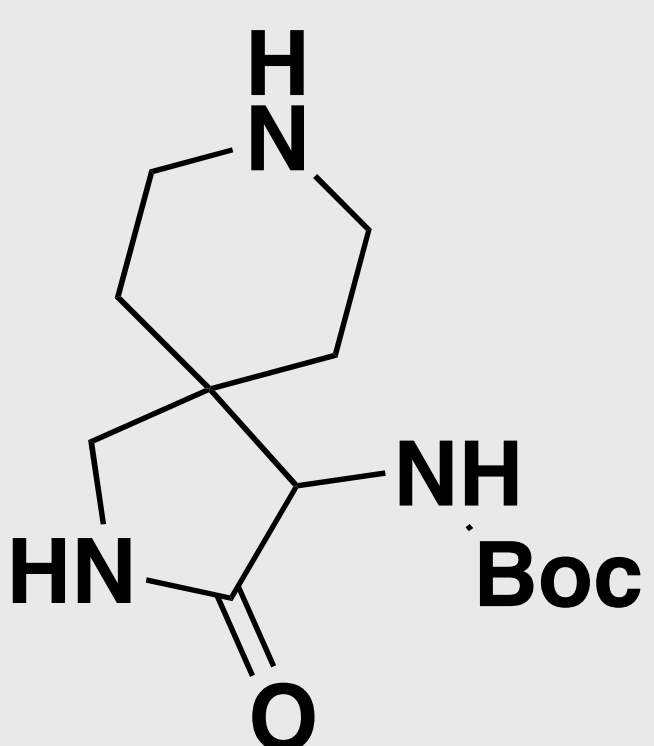

N300-26666762

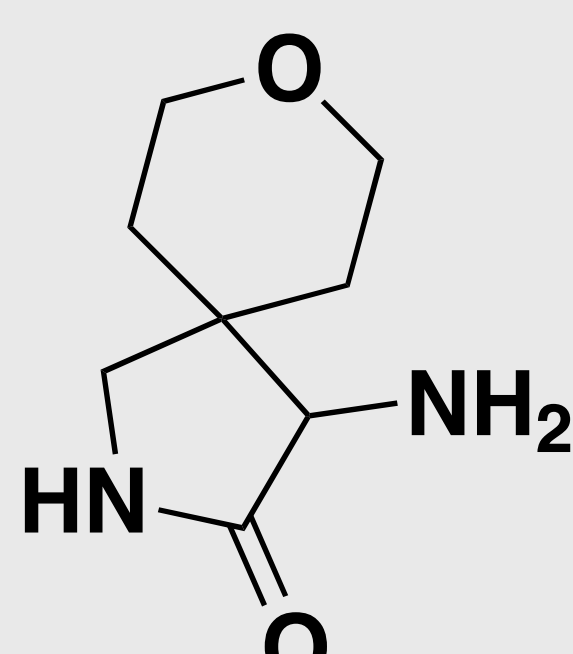

EN300-310254<smiles>CC1(C(=O)[O-])CC2(CNC(=O)C2=O)C1</smiles>

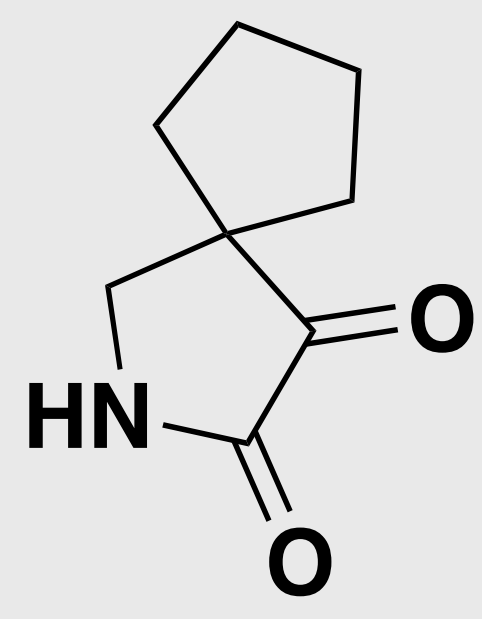

EN300-6497778

EN300-6497774

EN300-7440815

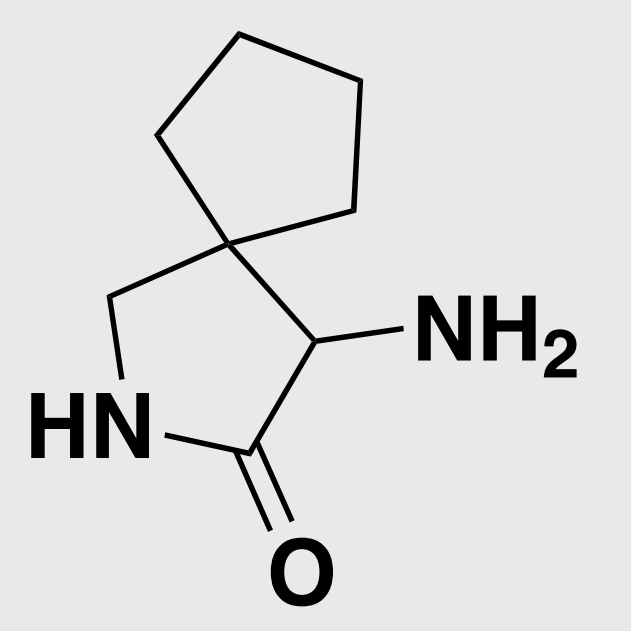

EN300-784920

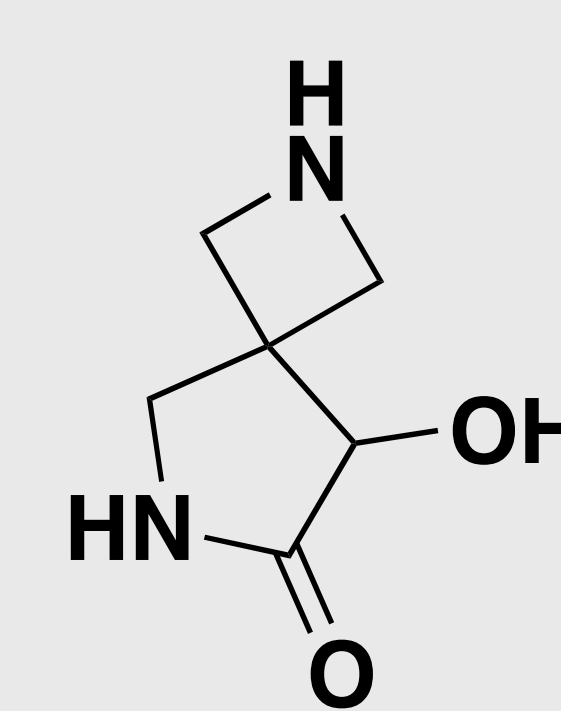

EN300-7439810

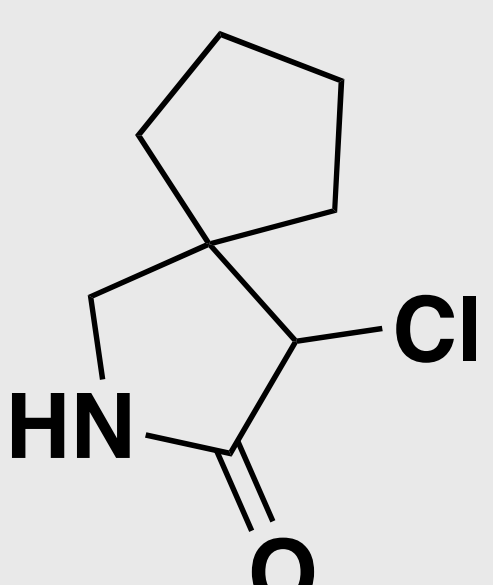

EN300-26666767

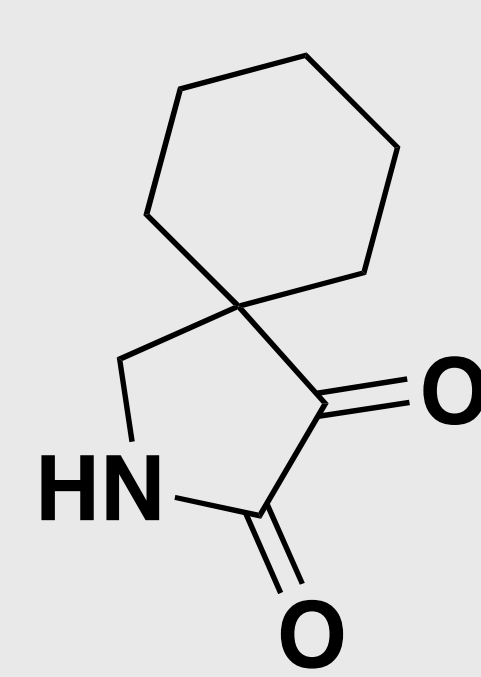

EN300-6497779<smiles>NC1C(=O)NCC12CNC2(O)c1ccccc1</smiles>

EN300-7439939

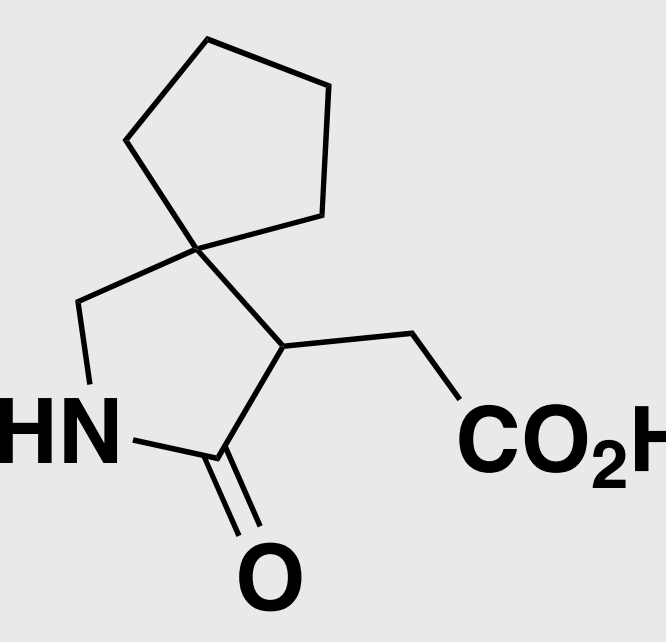

EN300-26666768

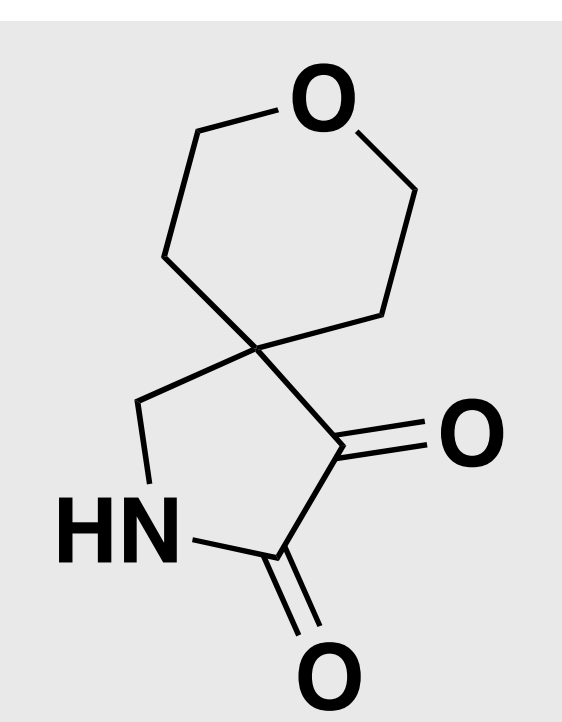

EN300-253382<smiles>CC(C)(C)OC(=O)C1C(=O)NCC12CNC2</smiles>

EN300-26666766

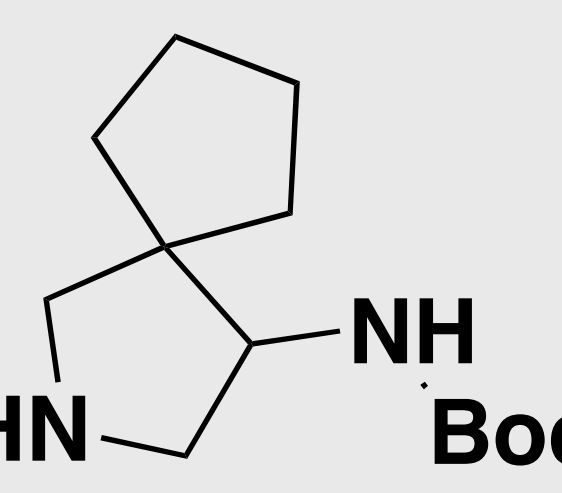

EN300-7096138

\section{Contact}

